Breite Behandlungspalette

\section{Vielfalt bei der MS-Therapie}

Ob täglich Tabletten oder Injektionen nur alle zwei Wochen: Für Patienten mit leichter bis moderat aktiver Multipler Sklerose gibt es jetzt eine breite Palette von Therapien und Applikationsformen.

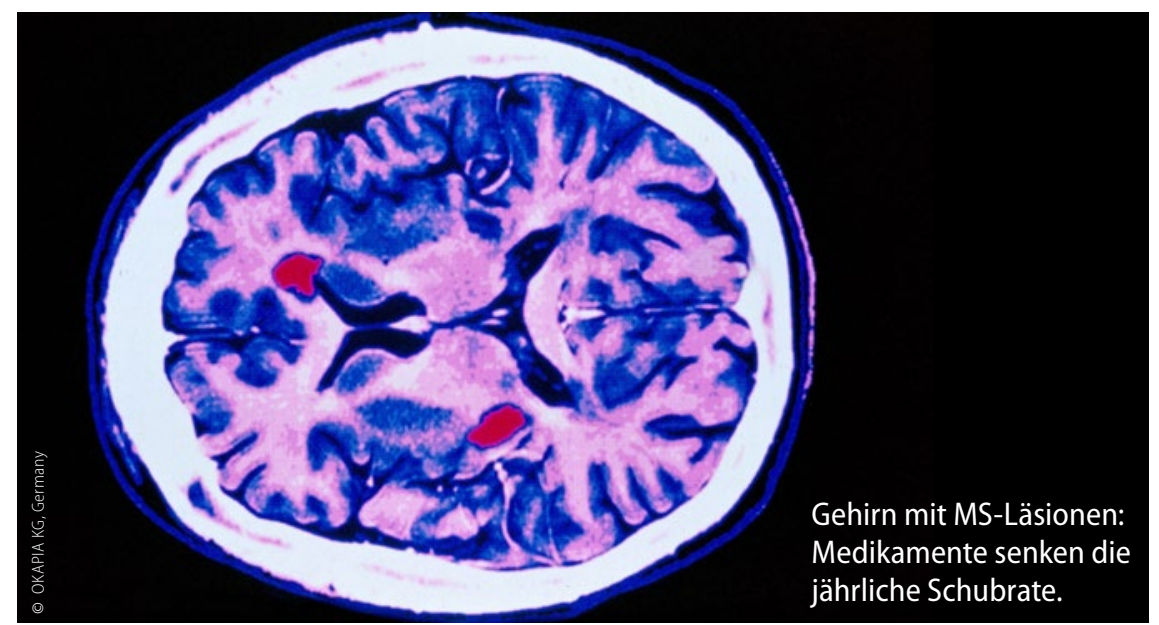

Bei der Basistherapie gegen die schubförmige Multiple Sklerose (MS) haben Ärzte und Patienten inzwischen die Qual der Wahl: Neben den älteren injizierbaren Medikamenten stehen seit Kurzem auch neue oral anwendbare Immunmodulatoren zur Verfügung.

Die Therapien unterscheiden sich vor allem hinsichtlich der Applikationsform und -frequenz. Auch das Nebenwirkungsspektrum der Substanzen ist unterschiedlich. Mit Langzeitdaten über mittlerweile mehr als 20 Jahre gibt es die besten Erfahrungen zu Beta-Interferonen und Glatirameracetat. Die häufigsten Nebenwirkungen sind Lokalreaktionen und grippeähnliche Symptome. Aufgrund der guten Verträglichkeit wurden Beta-Interferone auch zur Therapie während der Schwangerschaft freigegeben.

Sowohl Beta-Interferone als auch Glatirameracetat sind bereits bei einem klinisch isolierten Syndrom (CIS) zugelassen, ebenso bei einem hohen Risiko, eine manifeste MS zu entwickeln. Einige der Beta-Interferone sind auch bei Patienten mit sekundär-progredienter MS mit aufgesetzten Schüben indiziert.

\section{Vergleichbare Wirksamkeit}

Vergleichsstudien zwischen den einzelnen Interferonen und Glatirameracetat deuten auf eine ähnlich gute Wirksamkeit. Derzeit erhältlich sind die Interferone beta-1a (Avonex ${ }^{\oplus}$, Rebif ${ }^{\star}$ ) und beta-1b (Betaferon", Extavia ${ }^{\circ}$ ). Sie unterscheiden sich in der Applikationsform (i.m. und s.c.) sowie in der Applikationsfrequenz (einmal täglich bis einmal wöchentlich). Neu hinzugekommen ist vor einem Jahr Peginterferon beta1a (Plegridy $)$. Damit genügt eine subkutane Injektion alle zwei Wochen. In der Studie ADVANCE ließ sich mit dem Präparat die jährliche Schubrate im Vergleich zu Placebo um 36\% reduzieren. Auch mit einer neuen 40-mg-Formulierung von Glatirameracetat (Copaxone ${ }^{\circ}$ ) sind weniger Injektionen nötig - nur noch drei pro Woche. In der Studie GLACIER kam es dabei nur noch halb so oft zu injektionsbedingten Nebenwirkungen wie bei einer täglichen Applikation von $20 \mathrm{mg}$.
Medikamente zur MS-Basistherapie

Mit Langzeitdaten über mittlerweile mehr als 20 Jahre gibt es die besten Erfahrungen zu Beta-Interferonen und Glatirameracetat. Alternativen in Tablettenform sind mit Teriflunomid und Dimethylfumarat in den vergangenen zwei Jahren zur Basistherapie bei MS hinzugekommen.

Mit Teriflunomid (Aubagio ${ }^{\circledast}$ ) und Dimethylfumarat (Tecfidera ${ }^{\circ}$ ) sind in den vergangenen zwei Jahren Alternativen in Tablettenform zur Basistherapie bei Patienten mit MS hinzugekommen. Teriflunomid konnte in den beiden Zulassungsstudien TEMSO und TOWER die jährliche Schubrate im Vergleich zu Placebo um $32 \%$ bzw. um $36 \%$ reduzieren.

Und in der Studie TOPIC entwickelte sich unter Teriflunomid eine klinisch manifeste MS bei CIS-Patienten im Laufe von zwei Jahren nach den neuen McDonald-Kriterien nur rund halb so oft wie unter Placebo.

Der Immunmodulator braucht auch den Vergleich mit älteren Basistherapeutika nicht zu scheuen: In der Studie TENERE ergab sich eine ähnliche Wirksamkeit wie mit Interferon beta-1a s.c.

\section{Gute Sicherheitsdaten}

Dimethylfumarat (DMF) ist bereits seit Februar vergangenen Jahres erhältlich. In den beiden Zulassungsstudien DEFINE und CONFIRM ließ sich die jährliche Schubrate mit dem Medikament im Vergleich zu Placebo um 53\% bzw. um 44\% senken. Und in der noch laufenden Extensionsstudie ENDORSE waren 60\% der Patienten unter einer Therapie mit Dimethylfumarat auch nach fünf Jahren noch schubfrei.

Sowohl mit Dimethylfumarat als auch mit Teriflunomid ließ sich in Studien eine signifikante Verzögerung der Behinderungsprogression bei MS-Patienten nachweisen. Außerdem: Für beide Substanzen liegen Sicherheitsdaten von mehreren Tausend Patienten vor - zum Teil über zwölf Jahre hinweg. Häufige und meist transiente Nebenwirkungen sind danach bei der Behandlung mit Dimethylfumarat Flushes und gastrointestinale Beschwerden, bei einer Therapie mit Teriflunomid sind es reversible Haarausdünnung und Leberenzymerhöhungen. (ThomasMüller)

www.aerztezeitung.de 